\title{
Mechanical Reshaping of Inorganic Nanostructures with Weak Nanoscale Forces
}

Sarah M. Rehn ${ }^{1}$, Theodor M. Gerrard-Anderson ${ }^{1}$, Liang Qiao ${ }^{1}$, Qing Zhu', Geoff Wehmeyer ${ }^{2}$, Matthew R. Jones $* 1,3$

${ }^{1}$ Department of Chemistry, Rice University, Houston, Texas 77005, United States.

${ }^{2}$ Department of Mechanical Engineering, Rice University, Houston, Texas 77005, United States.

${ }^{3}$ Department of Materials Science and NanoEngineering, Rice University, Houston, Texas 77005, United States.

*Corresponding author. Email: mrj@rice.edu

Keywords: nanoplate, post-synthetic modification, nanomechanics, deformation, curvilinear, nanostructure

\section{Experimental Methods}

\section{Chemicals and Materials}

All chemicals were used as purchased with no further purification or processing. Trisodium citrate $\left(\mathrm{Na}_{3} \mathrm{C}_{6} \mathrm{H}_{5} \mathrm{O}_{7} \bullet 2 \mathrm{H}_{2} \mathrm{O}, 99.0 \%\right.$ ACS Reagent), silver nitrate $\left(\mathrm{AgNO}_{3}\right.$, $99.9999 \%$ trace metals basis), hydrogen peroxide $\left(\mathrm{H}_{2} \mathrm{O}_{2}, 30 \% \mathrm{w} / \mathrm{w}\right.$ in water ACS reagent with inhibitor), sodium borohydride $\left(\mathrm{NaBH}_{4}, 99.99 \%\right.$ trace metals basis), and L-ascorbic acid $\left(\mathrm{C}_{6} \mathrm{H}_{8} \mathrm{O}_{6}, 99.0 \%\right.$ bioXtra $)$ were purchased from Sigma Aldrich. Acetonitrile $\left(\mathrm{C}_{2} \mathrm{H}_{3} \mathrm{~N}\right.$, ChromAR) was purchased from Macron. The PEG-thiol molecule used for nanoprism stabilization, $\mathrm{HS}-\mathrm{C}_{11}-(\mathrm{PEG})_{6}-\mathrm{COOH}$, was purchased from Prochimia Surfaces. Oleic acid capped iron oxide nanospheres $(15 \mathrm{~nm})$ in chloroform were purchased from Ocean NanoTech. 


\section{Preparation of Ag Nanoplate Seeds}

Ag nanoprism seeds were prepared following a previously published protocol [1]. Seeds were prepared in an aqua regia-cleaned scintillation vial by combining fresh nanopure water $(20 \mathrm{~mL})$, trisodium citrate $(1.2 \mathrm{~mL}, 75 \mathrm{mM}), \mathrm{AgNO}_{3}(0.020 \mathrm{~mL}, 100 \mathrm{mM})$, and $\mathrm{H}_{2} \mathrm{O}_{2}$ $(0.048 \mathrm{~mL}, 30 \% \mathrm{w} / \mathrm{w})$ under vigorous stirring. To this, a freshly prepared solution of $\mathrm{NaBH}_{4}(0.120 \mathrm{~mL}, 100 \mathrm{mM})$ was injected, and the reaction progressed over a 30 -minute time period where the solution initially turned yellow and progressed through several color stages that finally ended at a cerulean blue. The nanoprism seeds were stored on a benchtop at room temperature.

\section{Seeded Growth of High Aspect Ratio Silver Nanoplates}

The growth of high aspect ratio silver nanoprisms was adapted from a previously published report [2]. To an aqua-regia cleaned scintillation vial in an ice bath, fresh nanopure water $(10 \mathrm{~mL})$, acetonitrile $(5 \mathrm{~mL})$, trisodium citrate $(0.200 \mathrm{~mL}, 75 \mathrm{mM})$, and ascorbic acid $(0.100 \mathrm{~mL}, 100 \mathrm{mM})$ were added under vigorous stirring. Meanwhile, an aliquot of the previously prepared seeds was subjected to centrifugation (10 minutes, $20133 \mathrm{rcf}$ ) to remove unreacted precursors. After the spin cycle, the supernatant was removed and the pelleted nanoprisms were concentrated $5 \mathrm{x}$ via resuspension in fresh nanopure water. These concentrated silver nanoprism seeds were immediately added to the reaction vial $(0.035$ $\mathrm{mL})$, after which $\mathrm{AgNO}_{3}(0.120 \mathrm{~mL}, 100 \mathrm{mM})$ was injected. This slow growth reaction proceeded to stir vigorously in the ice bath for approximately two hours, yielding a very pale blue solution, before being moved to the refrigerator to finish growth overnight, after which that large silver nanoprisms had visibly settled out of solution as a grey precipitate. Upon disturbing the precipitate, the solution appeared to be blue with a grey pearlescent quality, indicating the presence of the large, light scattering nanoprisms. Grown nanoprisms were then stabilized before further characterization by incubating an aliquot 
of the as-synthesized nanoprisms with a PEG-thiol molecule $(0.100 \mathrm{mM})$ at $4{ }^{\circ} \mathrm{C}$ under shaking overnight.

\section{Preparation of TEM and AFM Samples}

Iron oxide nanospheres $(0.001 \mathrm{~mL})$ were freshly diluted into toluene $(15 \mathrm{~mL})$. Large silver nanoprisms were spun down (1 min) with a tabletop centrifuge, supernatant was removed, and the nanoparticle pellet was resuspended with an equal volume of fresh nanopure water. To prepare a TEM sample, diluted iron nanoparticles $(0.0035 \mathrm{~mL})$ were drop cast onto a TEM grid. After the grid dried, washed nanoprisms $(0.0065 \mathrm{~mL})$ were drop cast onto the same grid and dried under vacuum. All wide field BF, DF, and SAED data were obtained with a JEOL 1230 High Contrast TEM operated at $80 \mathrm{kV}$. High resolution TEM data were obtained with a JEOL 2100 FEG TEM operated at $200 \mathrm{kV}$. All size analysis from TEM images was done using ImageJ. To prepare AFM samples, diluted iron nanoparticles $(0.015 \mathrm{~mL})$ were drop cast onto a mica disc and dried under vacuum. After drying, washed nanoprisms $(0.030 \mathrm{~mL})$ were drop cast onto the same disc and dried under vacuum. All AFM data were obtained with a Park NX20 AFM using tapping mode.

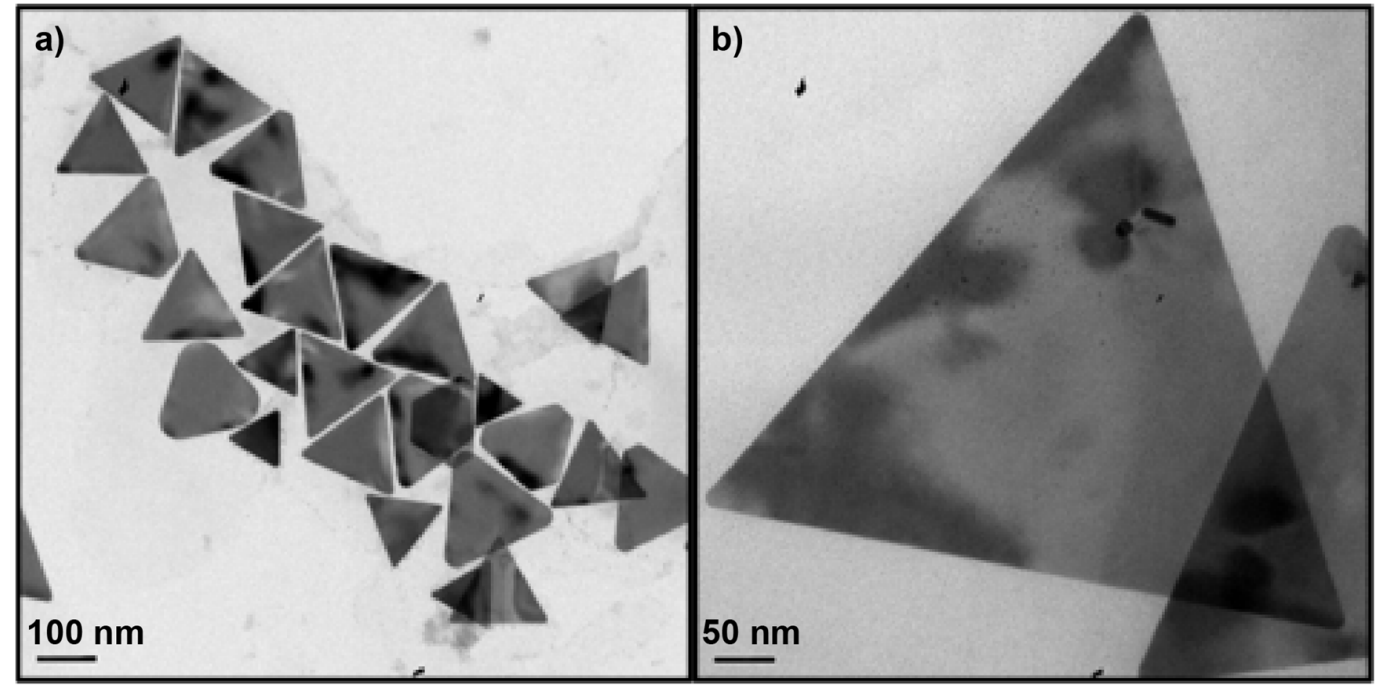

Figure S1. Representative BF TEM images of a) silver nanoprism seeds and b) a grown, high aspect ratio silver nanoprism. 


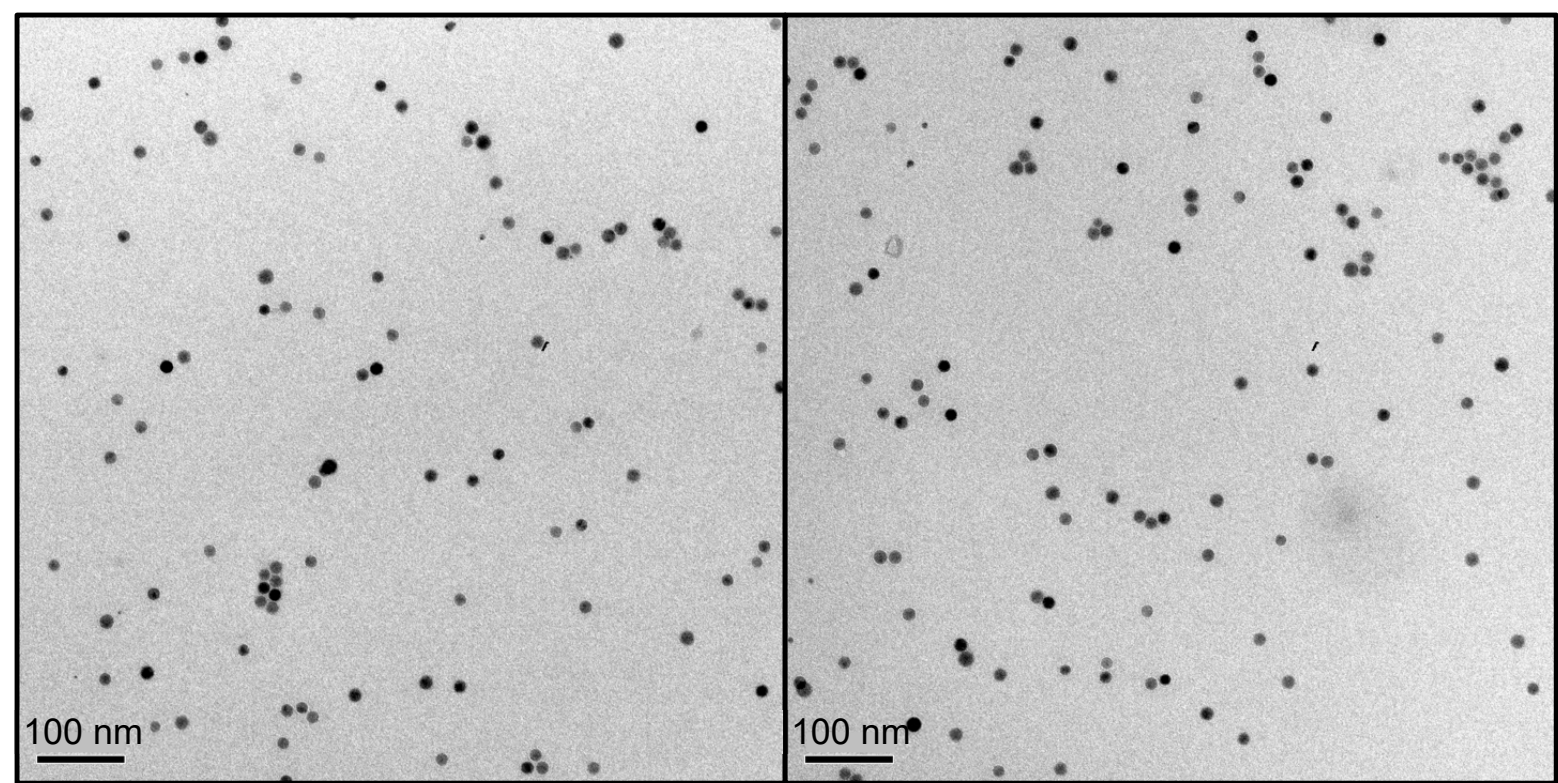

Figure S2. Representative BF TEM images of iron oxide nanospheres obtained from Ocean NanoTech. Size analysis of TEM images with ImageJ revealed the size distribution to be $15 \pm 1 \mathrm{~nm}(\mathrm{n}=159)$. Iron oxide nanoparticles were used as templates due to their high degree of shape and size uniformity.

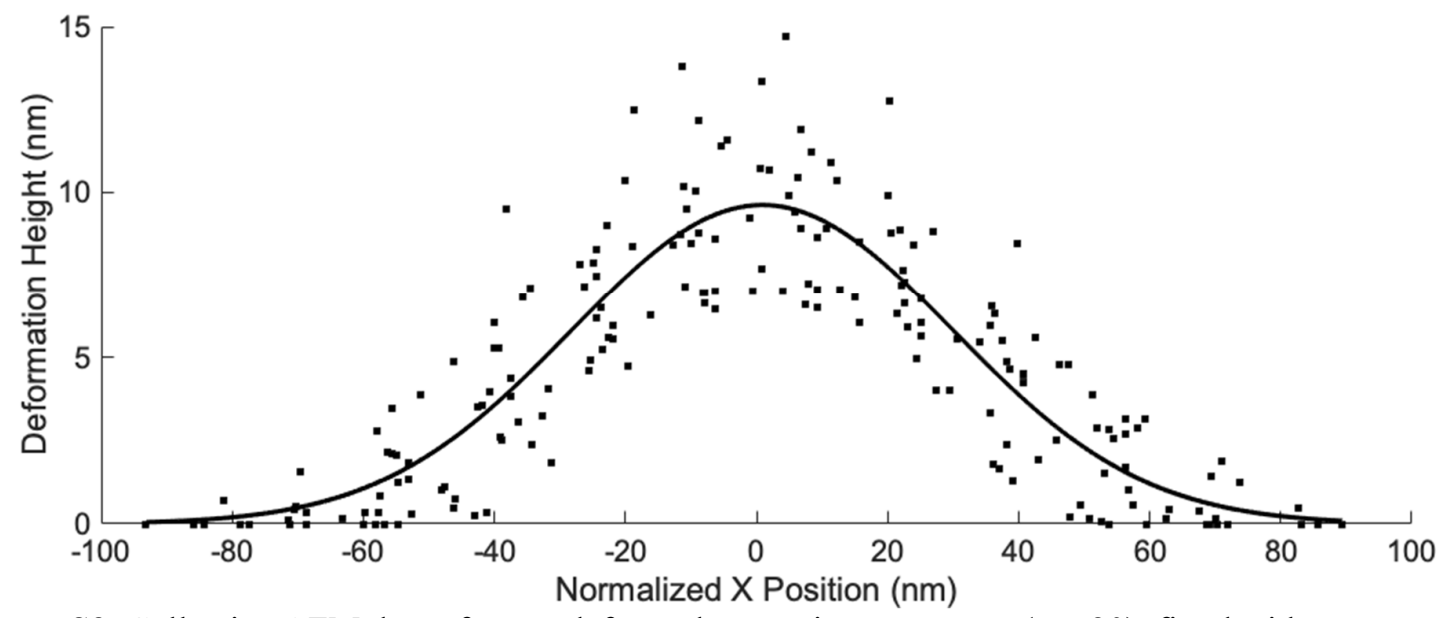

Figure S3. Collective AFM data of many deformed nanoprism structures $(n=20)$, fitted with to a Gaussian. The FWHM of these data is $66 \mathrm{~nm}$, which matches well with the bend contour diameter data from TEM analysis. Nanoprism thickness was also measured with AFM and found to be $8.8 \pm 1.3 \mathrm{~nm}$ (n $=24$ ). These data can be found normalized in the maintext Fig. 3a. 


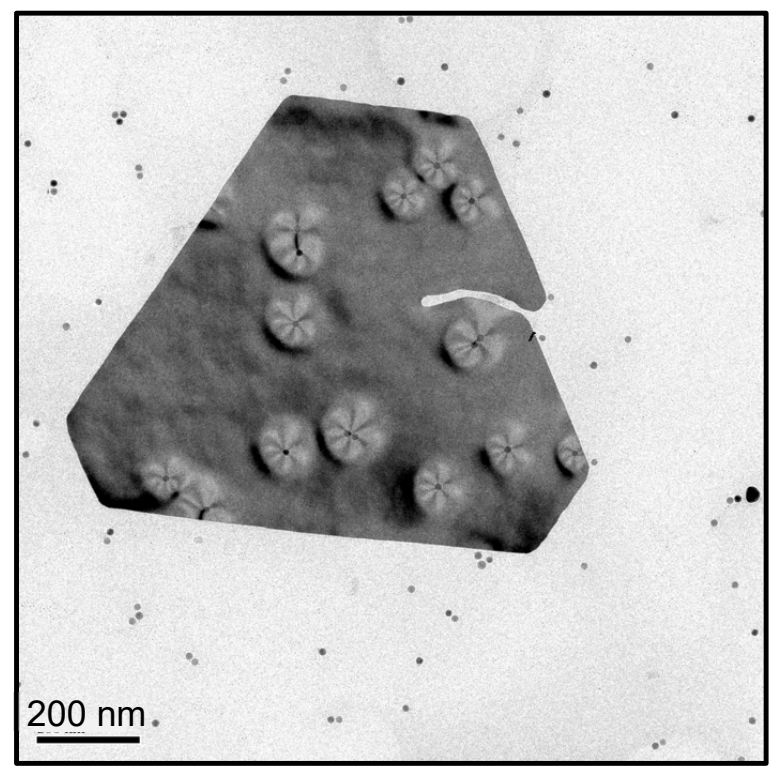

Figure S4. Wide field TEM image of the particle used for dark field analysis in main text Fig 2.

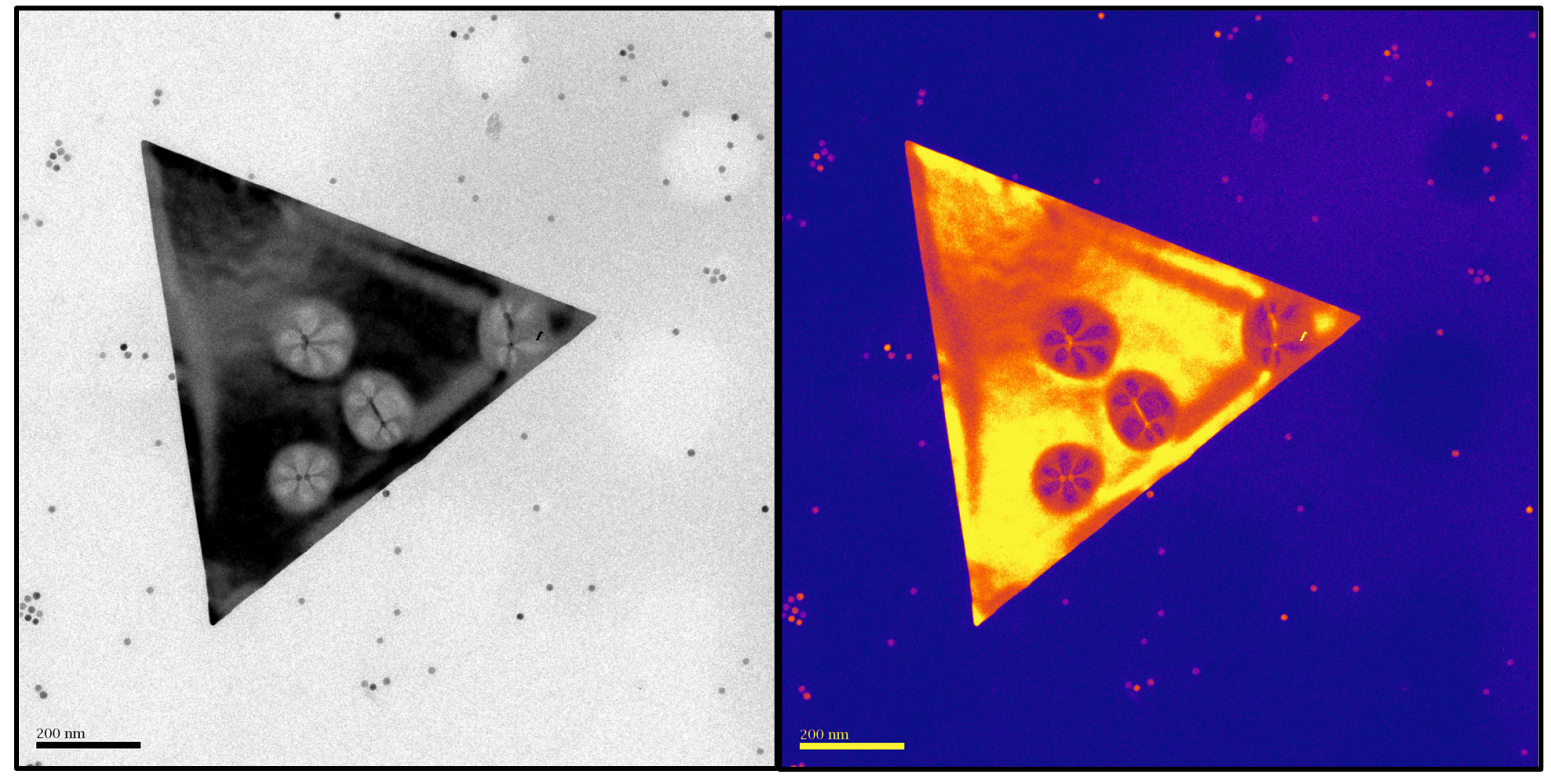

Figure S5. Example TEM image to demonstrate the colormap applied to images in main text Fig 4. The colormap was applied to help highlight differences in contrast that may be difficult to decipher in the grayscale image. Four-color map was directly applied to image with Adobe Photoshop. 


\section{Modification of Kirchhoff-Love Plate Theory for Plastic Deformation}

In order to calculate the strain energy for a deforming nanoplate we used Kirchhoff-Love plate theory, which is normally only valid for elastic deformation. Therefore, we made a modification that would allow strain energy to be calculated for sections undergoing plastic deformation as well as elastic deformation [3]. This modification was required because of the large deformations for which we wanted to be able to calculate strain energies.

We began with a relatively simple solution to Kirchhoff-Love plate theory; Fig. S6 illustrates the case of an axisymmetric plate with a concentrated force applied to its centre and restrained at a circular boundary distance $(a)$ from the center point. This analytical model accurately captures the deformed regions observed on the silver nanoplates, as the circular plate geometry matches the circular symmetry of the observed deformation. The point load models the force of the spherical template particle against the bottom of the plate; note that the point load assumption is reasonable because the radius of the particle is an order of magnitude less than the radius of the total deforming area. The clamped boundary condition is valid because the circular deformed region is continuous with the surrounding plate area, which is adhered to the substrate via $\mathrm{VdW}$ forces. The surrounding substrate can therefore neither translate or rotate, and the transition from this region to the deformed region (the boundary) is fixed. Cylindrical polar coordinates are natural for this solution and the displacement field has the following form: 


$$
w=\frac{w_{\max }}{a^{2}}\left(2 r^{2} \ln \left(\frac{r}{a}\right)+a^{2}-r^{2}\right)
$$

Where $w$ is the displacement in the $z$ axis of the neutral plane of the plate at any point $r$. The neutral plane is defined as the plane spanning the plate midway through its thickness $(\mathrm{z}=0)$ which experiences no stress. $w_{\max }$ is the displacement of the centre of the plate at the location of the concentrated deforming force $(r=0), a$ is the distance between the centre of the plate and the restrained edge, and $r$ is the radial coordinate. The $\theta$ coordinate does not appear in the displacement field owing to the axial symmetry of the plate geometry (Fig. S6).

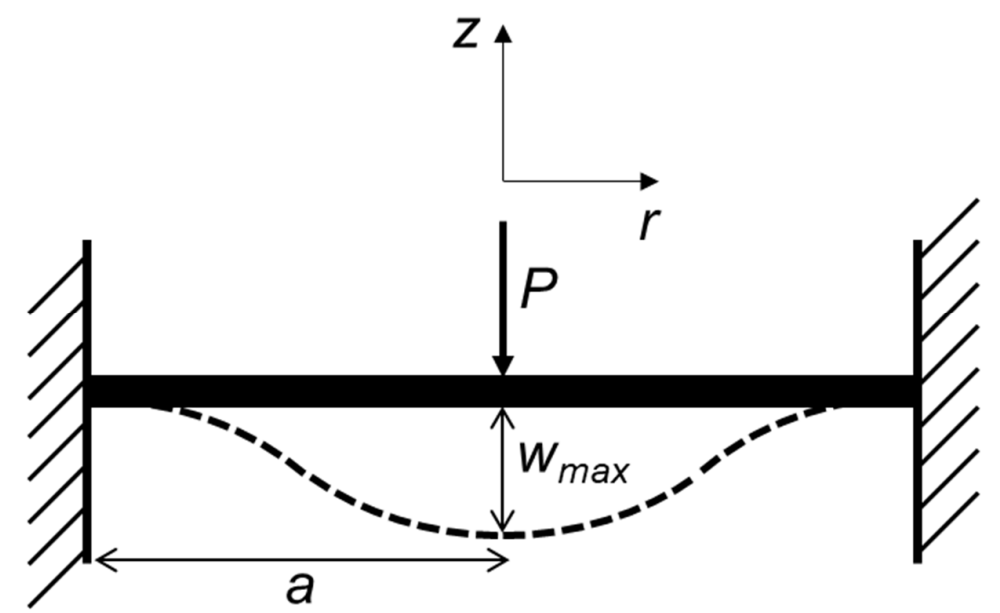

Figure S6. Diagram of the axially symmetric circular plate with a restrained edge and concentrated force acting on its centre. The solid line represents the undeformed plate and the dashed line the deformed plate. $P$ is the concentrated central force, $w_{\max }$ is the maximum displacement of the plate, and $a$ is the radius of the plate. The coordinate axes are shown.

The equation for this system of elastic strain energy per unit volume, $\frac{U_{\text {elastic }}}{V}$, is as follows: 


$$
\frac{U_{\text {elastic }}}{V}=\frac{1}{2}\left(\varepsilon_{r} \sigma_{r}+\varepsilon_{\theta} \sigma_{\theta}\right)
$$

Where $\varepsilon_{r}, \varepsilon_{\theta}, \sigma_{r}$, and $\sigma_{\theta}$ are the strains and stresses in the radial and polar directions, respectively. Kirchhoff-Love plate theory assumes plane stress conditions, so $\varepsilon_{z}$ and $\sigma_{z}$ are zero and so do not make an appearance. Additionally, shear strains are zero owing to the choice of coordinate system. This equation is valid until the point where the material yields. For strains beyond the yield strain, assuming fully plastic behaviour, the following equation is used:

$$
\frac{U_{\text {plastic }}}{V}=\left(\varepsilon_{r}-\varepsilon_{r y}\right) \sigma_{r y}+\left(\varepsilon_{\theta}-\varepsilon_{\theta y}\right) \sigma_{\theta y}
$$

Where $\varepsilon_{r y}, \varepsilon_{\theta y}, \sigma_{r y}$, and $\sigma_{\theta y}$ are the strains and stresses at yielding. The lack of a $\frac{1}{2}$ term is due to stress remaining constant for increased strains after yielding. The area under the stress-strain curve is defined by a rectangle so is merely the product of the stress and strain (Fig. S7).

The total strain energy per unit volume for a point that has yielded is then found by combining equations (2) and (3):

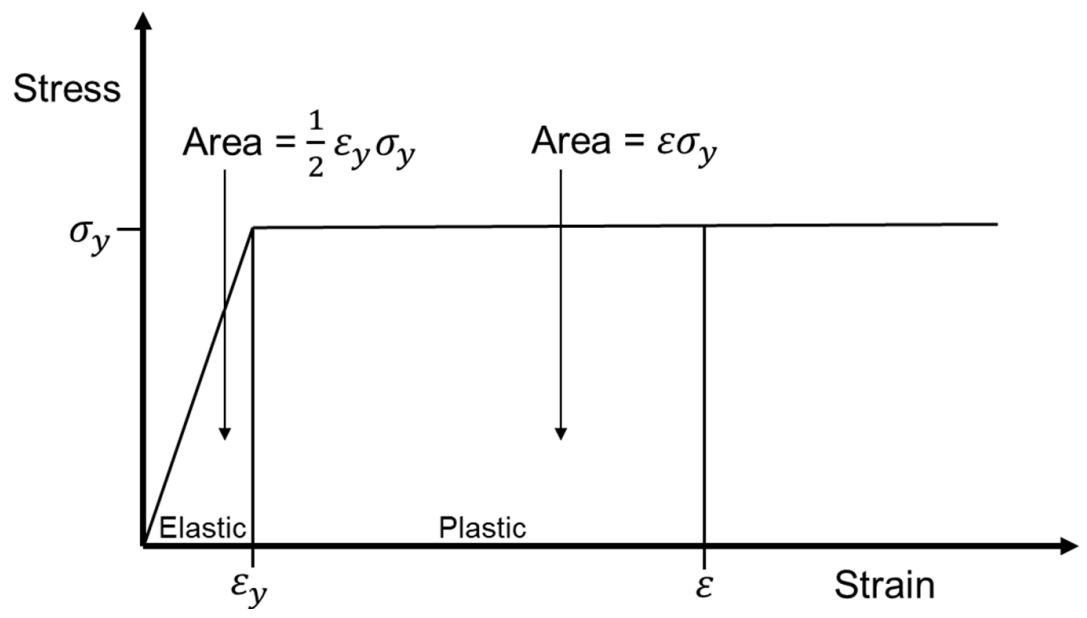

Figure S7. Stress-strain curve illustrating elastic-fully plastic behaviour. The change in the way the area under the graph, and therefore the strain energy, is calculated for regions of elastic deformation and nlastic deformation is shown. 


$$
\frac{U_{e y+p}}{V}=\left(\varepsilon_{r}-\frac{1}{2} \varepsilon_{r y}\right) \sigma_{r y}+\left(\varepsilon_{\theta}-\frac{1}{2} \varepsilon_{\theta y}\right) \sigma_{\theta y}
$$

Where $\frac{U_{e y+p}}{V}$ is the combined elastic and plastic contributions to strain energy per unit volume at a point where yielding has already occurred. In order to find $\varepsilon_{r y}, \varepsilon_{\theta y}, \sigma_{r y}$, and $\sigma_{\theta y}$, we need to define the yield criterion for this system. The Von Mises yield criterion is the most accurate for ductile metals, such as silver, so it will be used to define yielding in this system [4]:

$$
\sqrt{\sigma_{r}^{2}+\sigma_{\theta}^{2}-\sigma_{r} \sigma_{\theta}}=\sigma_{y}
$$

Where $\sigma_{y}$ is the yield stress for the material in a uniaxial tensile test. The left-hand term represents the equivalent von Mises stress. Once the von Mises stress is equal to $\sigma_{y}$, yielding has occurred and plastic deformation begins. The relations between $\sigma_{r}$ and $\sigma_{\theta}$ and the displacement field in the elastic range for this system are shown below:

$$
\begin{gathered}
\sigma_{r}=-\frac{E z}{1-v^{2}}\left(\frac{d^{2} w}{d r^{2}}+\frac{v}{r} \frac{d w}{d r}\right) \\
\sigma_{\theta}=-\frac{E z}{1-v^{2}}\left(\frac{1}{r} \frac{d w}{d r}+v \frac{d^{2} w}{d r^{2}}\right)
\end{gathered}
$$

Where $E$ is the Young's modulus of the material, $z$ is the coordinate in the $z$ direction, and $v$ is Poisson's ratio. Carrying out the differentiations on equation (1) and substituting them into equations (6) and (7) yields:

$$
\sigma_{r}=-\frac{4 E z w_{\max }}{a^{2}\left(1-v^{2}\right)}\left((v+1) \ln \left(\frac{r}{a}\right)+1\right)
$$




$$
\sigma_{\theta}=-\frac{4 E z w_{\max }}{a^{2}\left(1-v^{2}\right)}\left((v+1) \ln \left(\frac{r}{a}\right)+v\right)
$$

Equations (8) and (9) can then be substituted into the Von Mises yield criterion (5) and then recombined with equations (8) and (9) separately to yield the formulae for $\sigma_{r y}$ and $\sigma_{\theta y}$ :

$$
\begin{aligned}
& \sigma_{r y}= \pm \frac{\sigma_{y}\left((v+1) \ln \left(\frac{r}{a}\right)+1\right)}{\sqrt{(v+1)^{2}\left(\ln \left(\frac{r}{a}\right)\right)^{2}+(1+2 v)(1+v) \ln \left(\frac{r}{a}\right)+v(v-1)+1}} \\
& \sigma_{\theta y}= \pm \frac{\sigma_{y}\left((v+1) \ln \left(\frac{r}{a}\right)+v\right)}{\sqrt{(v+1)^{2}\left(\ln \left(\frac{r}{a}\right)\right)^{2}+(1+2 v)(1+v) \ln \left(\frac{r}{a}\right)+v(v-1)+1}}
\end{aligned}
$$

The general elastic stress-strain relationship for this system is shown below:

$$
\varepsilon_{1}=\frac{\sigma_{1}-v \sigma_{2}}{E}
$$

Using equation (12) in combination with equations (8) and (9), expressions for the yield strains, $\varepsilon_{r y}$ and $\varepsilon_{\theta y}$, can be found:

$$
\begin{aligned}
& \varepsilon_{r y}= \pm \frac{\sigma_{y}\left((1-v)(v+1) \ln \left(\frac{r}{a}\right)+1-v^{2}\right)}{E \sqrt{(v+1)^{2}\left(\ln \left(\frac{r}{a}\right)\right)^{2}+(1+2 v)(1+v) \ln \left(\frac{r}{a}\right)+v(v-1)+1}} \\
& \varepsilon_{\theta y}= \pm \frac{\sigma_{y}(1-v)(v+1) \ln \left(\frac{r}{a}\right)}{E \sqrt{(v+1)^{2}\left(\ln \left(\frac{r}{a}\right)\right)^{2}+(1+2 v)(1+v) \ln \left(\frac{r}{a}\right)+v(v-1)+1}}
\end{aligned}
$$


In order to calculate strains beyond the yield strain, the equations for the radial and theta strains based on the displacement field are required. The strain-displacement relations are as follows:

$$
\begin{gathered}
\varepsilon_{r}=-z \frac{d^{2} w}{d r^{2}} \\
\varepsilon_{\theta}=-\frac{z}{r} \frac{d w}{d r}
\end{gathered}
$$

Carrying out the differentiations on equation (1) and substituting them into equations (15) and (16) yields:

$$
\begin{gathered}
\varepsilon_{r}=-\frac{4 z w_{\text {max }}}{3 a^{2}}\left(\ln \left(\frac{r}{a}\right)+1\right) \\
\varepsilon_{\theta}=-\frac{4 z w_{\text {max }}}{3 a^{2}} \ln \left(\frac{r}{a}\right)
\end{gathered}
$$

Now, equations (10), (11), (13), (14), (17), and (18) can, with careful attention paid to ensure the signs reflect reality, be substituted into equation (4) to yield:

$$
\begin{aligned}
& =\frac{U_{e y+p}}{V}-\frac{\sigma_{y}\left(1-v^{2}\right)\left((v+1)\left(\left(\ln \left(\frac{r}{a}\right)\right)^{2}+\ln \left(\frac{r}{a}\right)\right)+\frac{1}{2}\right)}{E\left((v+1)^{2}\left(\ln \left(\frac{r}{a}\right)\right)^{2}+(1+2 v)(1+v) \ln \left(\frac{r}{a}\right)+v(v-1)+1\right)} \\
& +\frac{8 z \sigma_{y} W_{\max }\left((v+1)\left(\left(\ln \left(\frac{r}{a}\right)\right)^{2}+\ln \left(\frac{r}{a}\right)\right)+\frac{1}{2}\right)}{3 a^{2} \sqrt{(v+1)^{2}\left(\ln \left(\frac{r}{a}\right)\right)^{2}+(1+2 v)(1+v) \ln \left(\frac{r}{a}\right)+v(v-1)+1}}
\end{aligned}
$$

While this equation describes the strain energy per unit volume at a point that has plastically deformed, there will be regions that are still in the process of elastic deformation. 
An equation that gives the strain energy per unit volume in an elastic region, $\frac{U_{\text {elastic }}}{V}$, can be found by substituting equations (8), (9), (17), and (18) into equation (2):

$$
\frac{U_{\text {elastic }}}{V}=\frac{16 w_{\text {max }}^{2} E}{\left(1-v^{2}\right) a^{4}} z^{2}\left((v+1)\left(\left(\ln \left(\frac{r}{a}\right)\right)^{2}+\ln \left(\frac{r}{a}\right)\right)+\frac{1}{2}\right)
$$

Now that expressions for the strain in areas of both plastic and elastic deformation, equations (19) and (20) respectively, have been obtained, they can be integrated over the total volumes experiencing plastic and elastic deformation respectively to attain $U_{\text {total }}$. Given that the system is axisymmetric, the function delineating the region where the deformation is elastic (closer to the neutral surface of the plate) from the region where deformation is plastic (closer to the top or bottom of the plate), will return a $z$ coordinate for a given $r$ coordinate and exclude $\theta$. This two-dimensional "yield line" can be found by rearranging the combination of equations (5), (8), and (9):

$$
\begin{aligned}
& z_{y}(r)= \pm \frac{\sigma_{y} a^{2}\left(1-v^{2}\right)}{4 E} w_{\max } \\
&+\left((v+1)^{2}\left(\ln \left(\frac{r}{a}\right)\right)^{2}\right. \\
&\left.+(1+2 v)(1+v) \ln \left(\frac{r}{a}\right)+v(v-1)+1\right)^{-\frac{1}{2}}
\end{aligned}
$$

This yield line function can then be used in the limits of the integral along the $z$ axis for $U_{\text {total }}$. For the region of plastic deformation, the limits will be $z_{y}$ and the surface of the plate $(t / 2)$, where $t$ is thickness. For the region of elastic deformation, the limits will be the neutral surface (0) and $z_{y}$ for the elastic region. Under the assumptions of KirchhoffLove plate theory, the material behaves the same way under tension and compression and stress is symmetric across the neutral plane. This allows for $U_{\text {total }}$ to be calculated by doubling the integration over the top half of the plate, rather than integrating over its full volume. This simplifies the final equation. After completing the integrals in the $z$ axis, the result must integrated through $\theta$ and $r$. The integral has the following form: 


$$
\begin{aligned}
& \int_{V_{\text {plastic }}} \frac{U_{\text {plastic }}}{V}+\int_{V_{\text {elastic }}} \frac{U_{\text {elastic }}}{V}=U_{\text {total }} \\
& =\int_{0}^{a} \int_{0}^{2 \pi}\left(\left(2 \int_{z_{y}(r)}^{\frac{t}{2}}-\frac{\sigma_{y}\left(1-v^{2}\right)\left((v+1)\left(\left(\ln \left(\frac{r}{a}\right)\right)^{2}+\ln \left(\frac{r}{a}\right)\right)+\frac{1}{2}\right)}{E\left((v+1)^{2}\left(\ln \left(\frac{r}{a}\right)\right)^{2}+(1+2 v)(1+v) \ln \left(\frac{r}{a}\right)+v(v-1)+1\right)}\right.\right. \\
& +\frac{8 z \sigma_{y} w_{\max }\left((v+1)\left(\left(\ln \left(\frac{r}{a}\right)\right)^{2}+\ln \left(\frac{r}{a}\right)\right)+\frac{1}{2}\right)}{\left.3 a^{2} \sqrt{(v+1)^{2}\left(\ln \left(\frac{r}{a}\right)\right)^{2}+(1+2 v)(1+v) \ln \left(\frac{r}{a}\right)+v(v-1)+1}\right) r d z} \\
& \left.+\left(2 \int_{0}^{z_{y}(r)} \frac{16 w_{\text {max }}^{2} E}{\left(1-v^{2}\right) a^{4}} Z^{2}\left((v+1)\left(\left(\ln \left(\frac{r}{a}\right)\right)^{2}+\ln \left(\frac{r}{a}\right)\right)+\frac{1}{2}\right)\right) r d z\right) d \theta d r
\end{aligned}
$$

Note the need to multiply the entire function by $r$ when integrating in cylindrical polar coordinates. The integrals in $z$ and $\theta$ are analytically solvable, however the integral in $r$ is not. This is reflected in the final form of the equation:

$$
\begin{gathered}
U_{\text {total }}=\pi \int_{0}^{a} r \alpha(r)\left(\frac{\sigma_{y}^{3} a^{2}\left(1-v^{2}\right)^{2}}{3 E^{2} w_{\max }}(\beta(r))^{-\frac{3}{2}}-\frac{2 t\left(1-v^{2}\right) \sigma_{y}^{2}}{E}(\beta(r))^{-1}\right. \\
\left.\quad+\frac{4 t^{2} \sigma_{y} w_{\max }\left(1-v^{2}\right)^{2}}{3 a^{2}}(\beta(r))^{-\frac{1}{2}}\right) d r \\
\alpha(r)=(v+1)\left(\left(\ln \left(\frac{r}{a}\right)\right)^{2}+\ln \left(\frac{r}{a}\right)\right)+\frac{1}{2} \\
\beta(r)=(v+1)^{2}\left(\ln \left(\frac{r}{a}\right)\right)^{2}+(1+2 v)(1+v) \ln \left(\frac{r}{a}\right)+v(v-1)+1
\end{gathered}
$$

As this is not analytically solvable, it must be integrated numerically to get $U_{\text {total }}$. For this we used the "integral" function in MATLAB.

However, when yield stresses exceed certain values, the entirety of the surface of the plate may not have yielded. In this case the integral must be split into five separate integrals 
using the coordinates of the two intersections between the yield line $\left(z_{y}\right)$ and the surface of the plate $t / 2, \mathrm{r}_{1}$ and $\mathrm{r}_{2}$, as new limits:

$$
\begin{aligned}
& r_{1}\left(Z_{y}=\frac{t}{2}\right) \\
& U_{\text {total }}=\pi \int_{0} r \alpha(r)\left(\frac{\sigma_{y}{ }^{3} a^{2}\left(1-v^{2}\right)^{2}}{3 E^{2} w_{\max }}(\beta(r))^{-\frac{3}{2}}\right) d r \\
& r_{2}\left(z_{y}=\frac{t}{2}\right) \\
& +\pi \int_{r_{1}\left(z_{y}=\frac{t}{2}\right)} r \alpha(r)\left(\frac{4 w_{\max ^{2}} E t^{3}}{3\left(1-v^{2}\right)^{2} a^{4}}\right) d r \\
& +\pi \int_{r_{2}\left(z_{y}=\frac{t}{2}\right)}^{a} r \alpha(r)\left(\frac{\sigma_{y}^{3} a^{2}\left(1-v^{2}\right)^{2}}{3 E^{2} w_{\max }}(\beta(r))^{-\frac{3}{2}}\right) d r \\
& +\pi \int_{0}^{a} r \alpha(r)\left(\frac{\sigma_{y}^{3} a^{2}\left(1-v^{2}\right)^{2}}{E^{2} w_{\max }}(\beta(r))^{-\frac{3}{2}}-\frac{t \sigma_{y}^{2}}{E}(\beta(r))^{-1}\right. \\
& \left.+\frac{t^{2} \sigma_{y} w_{\max }\left(1-v^{2}\right)^{2}}{3 a^{2}}(\beta(r))^{-\frac{1}{2}}\right) d r \\
& r_{1}\left(Z_{y}=\frac{t}{2}\right) \\
& +\pi \int_{0} r \alpha(r)\left(\frac{2 \sigma_{y}{ }^{3} a^{2}\left(1-v^{2}\right)^{2}}{3 E^{2} w_{\max }}(\beta(r))^{-\frac{3}{2}}\right. \\
& \left.-\frac{2 t\left(1-v^{2}\right) \sigma_{y}^{2}}{E}(\beta(r))^{-1}+\frac{4 t^{2} \sigma_{y} w_{\max }}{3 a^{2}}(\beta(r))^{-\frac{1}{2}}\right) d r \\
& +\pi \int_{r_{2}\left(z_{y}=\frac{t}{2}\right)}^{a} r \alpha(r)\left(\frac{2 \sigma_{y}{ }^{3} a^{2}\left(1-v^{2}\right)^{2}}{3 E^{2} w_{\max }}(\beta(r))^{-\frac{3}{2}}\right. \\
& \left.-\frac{2 t\left(1-v^{2}\right) \sigma_{y}^{2}}{E}(\beta(r))^{-1}+\frac{4 t^{2} \sigma_{y} w_{\max }}{3 a^{2}}(\beta(r))^{-\frac{1}{2}}\right) d r \\
& \alpha(r)=(v+1)\left(\left(\ln \left(\frac{r}{a}\right)\right)^{2}+\ln \left(\frac{r}{a}\right)\right)+\frac{1}{2} \\
& \beta(r)=(v+1)^{2}\left(\ln \left(\frac{r}{a}\right)\right)^{2}+(1+2 v)(1+v) \ln \left(\frac{r}{a}\right)+v(v-1)+1
\end{aligned}
$$

As this integral is also not analytically solvable, it must be integrated numerically to get $U_{\text {total }}$. In order to solve it the "integral" function in MATLAB. Using equations (23) and (24), the total strain energy of the system can be obtained for arbitrary input parameters. This can be 
compared with the VdW energy for the same input parameters to determine if the specified deformation can be brought about by the action of $\mathrm{VdW}$ forces between the plate and the substrate (see below).

\section{Calculation of Van der Waals Interaction Energy between a Nanoprism and Substrate}

The equation for the Van der Waals interaction energy per unit area between a plate of thickness $t$ and a substrate of effectively infinite thickness is as follows [5]:

$$
U_{A}=-\frac{A}{12 \pi}\left(\frac{1}{d^{2}}-\frac{1}{(d+t)^{2}}\right)
$$

Where $U_{A}$ is the VdW interaction energy per unit area, $A$ is the Hamaker constant for the system, and $d$ is the separation between the plate and the substrate. To calculate the overall Hamaker constant for the system of a triangular silver nanoplate interacting with a silicon substrate across a generic ligand layer containing a carbon chain we used the following equation [6]:

$$
A_{123} \approx\left(\sqrt{A_{11}}-\sqrt{A_{22}}\right)\left(\sqrt{A_{33}}-\sqrt{A_{22}}\right)
$$

Where $A_{123}$ is the Hamaker constant for medium 1 interacting with medium 3 through medium 2, and $A_{11}, A_{22}$, and $A_{33}$ are the Hamaker constants for the media 1,2 and 3 respectively. $A_{A g}=38.50 \times 10^{-20} \mathrm{~J}, A_{S i}=18.65 \times 10^{-20} \mathrm{~J}$, and $A_{\text {ligand }} \approx 5 \times 10^{-20} \mathrm{~J}[6]$. Therefore $A_{\text {Ag-ligand-Si }} \approx$

$8.26 \times 10^{-20} \mathrm{~J}$. In order to obtain the total energy of interaction we must multiply equation (24) by the area of the nanoplate. Assuming the triangular nanoplate has edges $1 \mu \mathrm{m}$ in length, the total area is $4.33 \times 10^{-13} \mathrm{~m}^{2}$. Incorporating this information into equation (24) we have:

$$
U_{V d W}=-\frac{8.26 \times 10^{-20}}{12 \pi}\left(\frac{1}{d^{2}}-\frac{1}{(d+t)^{2}}\right) \times 4.33 \times 10^{-13}
$$

Where $U_{v d w}$ is the total VdW interaction energy (in Joules) for a triangular silver nanoplate of thickness $t$ (in meters) with edges length 1 um, separated by a ligand layer of thickness $d$ (in meters) from a silicon substrate. 


\section{Estimating $\sigma_{y}$ from Experimental Data}

In an experimental scenario, due to the conservation of energy, the VdW interaction energy of a nanoplate must be equal to the strain energy of the deformation it is experiencing. Using this information, if we input the measured experimental parameters for $a(50 \mathrm{~nm})$, and $w_{\max }(15 \mathrm{~nm})$ and assume $v=0.37$ and that $\mathrm{E}=102 \mathrm{GPa}$ [7] (this is a mid-range value of $\mathrm{E}$ for silver nanoparticles, which varies between $\sim 90$ and $115 \mathrm{GPa}$ [8-11]), then a value for $\sigma_{y}$ can be extracted. Because the spacing (d) between the nanoplate and substrate is difficult to obtain, we calculated $\sigma_{y}$ for $\mathrm{d}=1,1.5$, and $2 \mathrm{~nm}$. The values of $\sigma_{y}$ are 708, 235, and $120 \mathrm{MPa}$ respectively. These $\sigma_{y}$ values are significantly elevated over the bulk $\sigma_{y}$ of $\sim 54 \mathrm{MPa}$. This is in agreement with previous experimental measurements of yield stress for silver nanoparticles which also demonstrate elevated values on the order of $1 \mathrm{GPa}[12]$.

\section{Comparing Van der Waals Interaction Energy and Strain Energy}

To visualize how VdW forces become strong enough to mechanically deform a triangular silver nanoplate of low enough thickness, we graphed the strain energy and VdW interaction energy against thickness on the same plot and observed the intersection of the two curves. In order to do this, experimentally observed input parameters were used: $a=50 \mathrm{~nm}, w_{\max }=15 \mathrm{~nm}$, as well as $d=2 \mathrm{~nm}$ (an estimate of ligand layer thickness) [13]. The material properties predicted above were used: $v=0.37, E=102 \mathrm{GPa}$, and $\sigma_{y}=120 \mathrm{MPa}$. Using these inputs, and leaving $t$ as a variable, we used equations (23) and (26) to generate the plot in the main text (Fig. 1a). This is shown again in Fig. S8

In order to demonstrate that Van der Waals forces are generally capable of deforming silver nanoplates, and that the input values shown above are not the only ones for which this is true, we have generated plots of the same form as main text Fig. 1, but with different yet still realistic input parameters (Fig. S9-S11). Using these it can be seen how the thickness at which a 
$1 \mu \mathrm{m}$ edge length triangular silver nanoplate becomes deformable by VdW forces changes based on the input parameters.

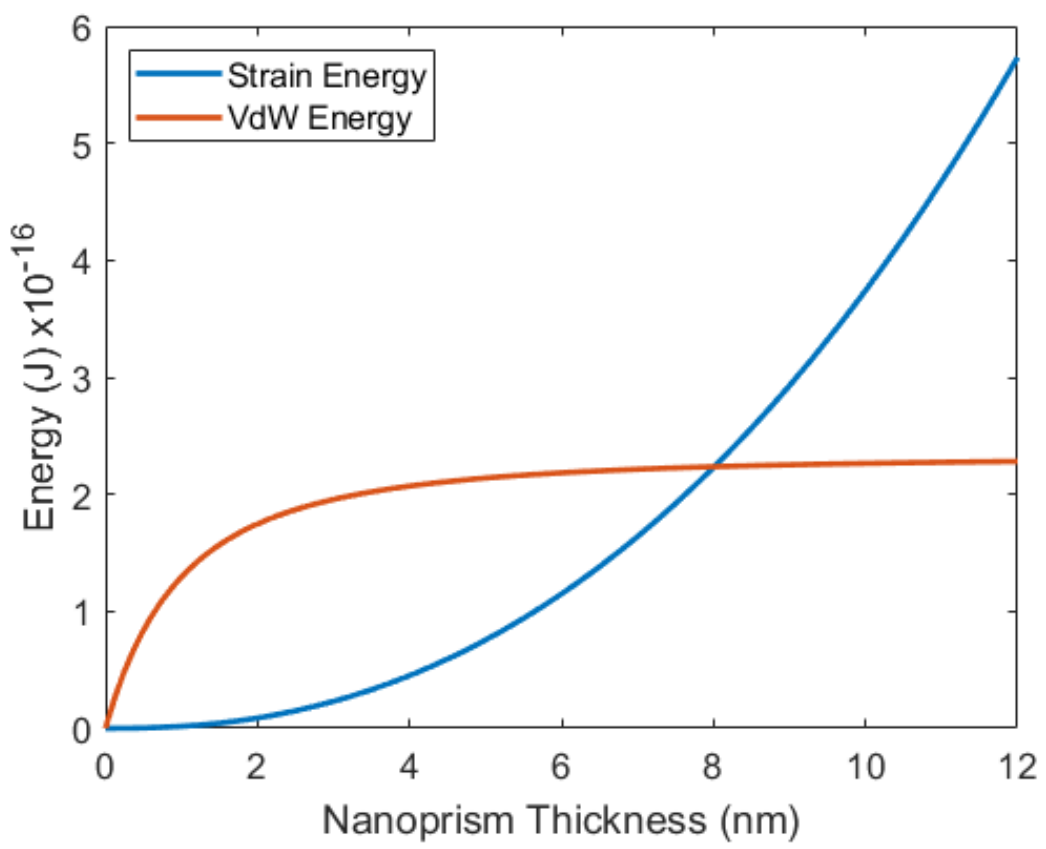

Figure S8. Plot of strain energy and VdW energy against nanoplate thickness. $W_{\max }=15 \mathrm{~nm}, a=50 \mathrm{~nm}, \mathrm{~d}=2 \mathrm{~nm}$

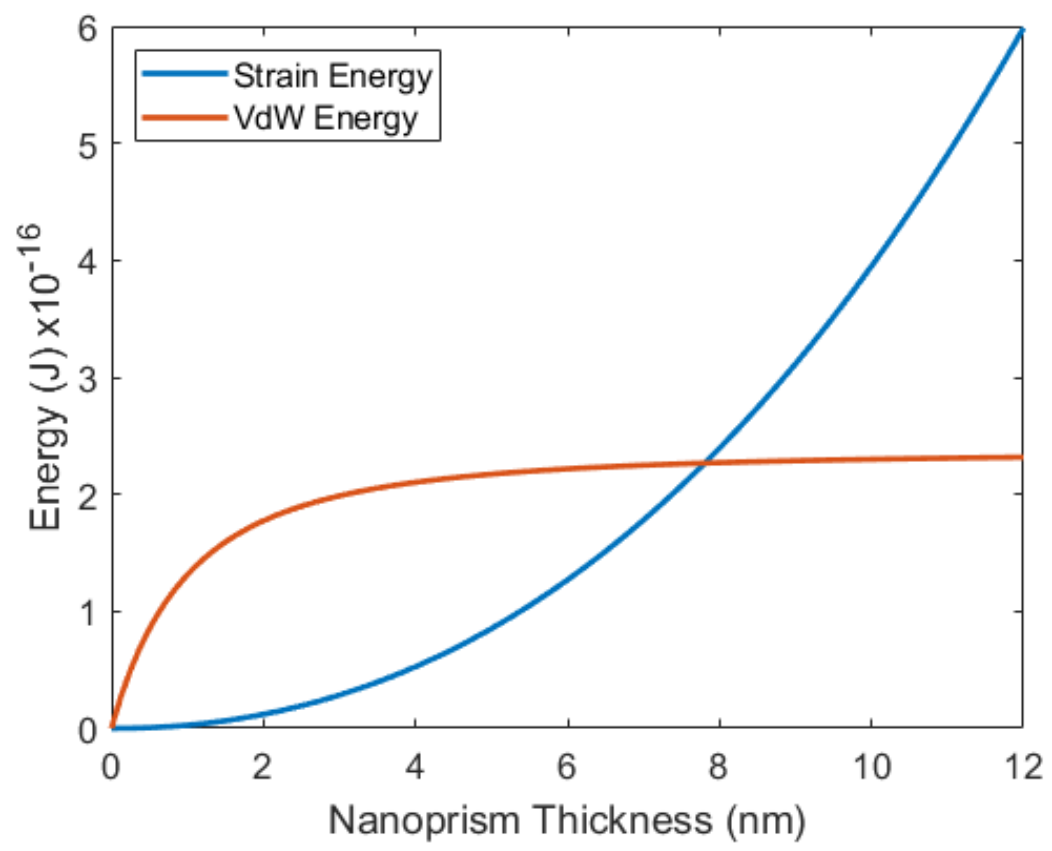

Figure S9. Plot of strain energy and VdW energy against nanoplate thickness. $\mathrm{W}_{\max }=15 \mathrm{~nm}, a=20 \mathrm{~nm}, \mathrm{~d}=2 \mathrm{~nm}$ 


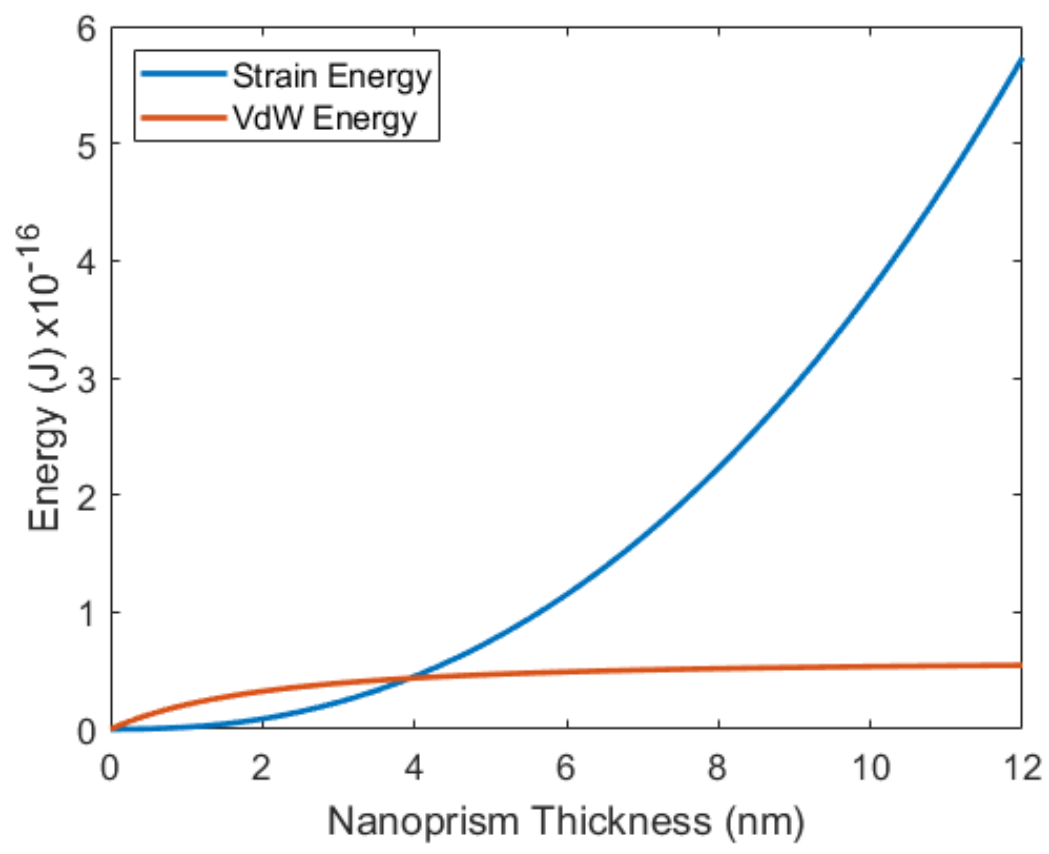

Figure S10. Plot of strain energy and VdW energy against nanoplate thickness. $W_{\max }=15 \mathrm{~nm}, a=50 \mathrm{~nm}, \mathrm{~d}=4 \mathrm{~nm}$

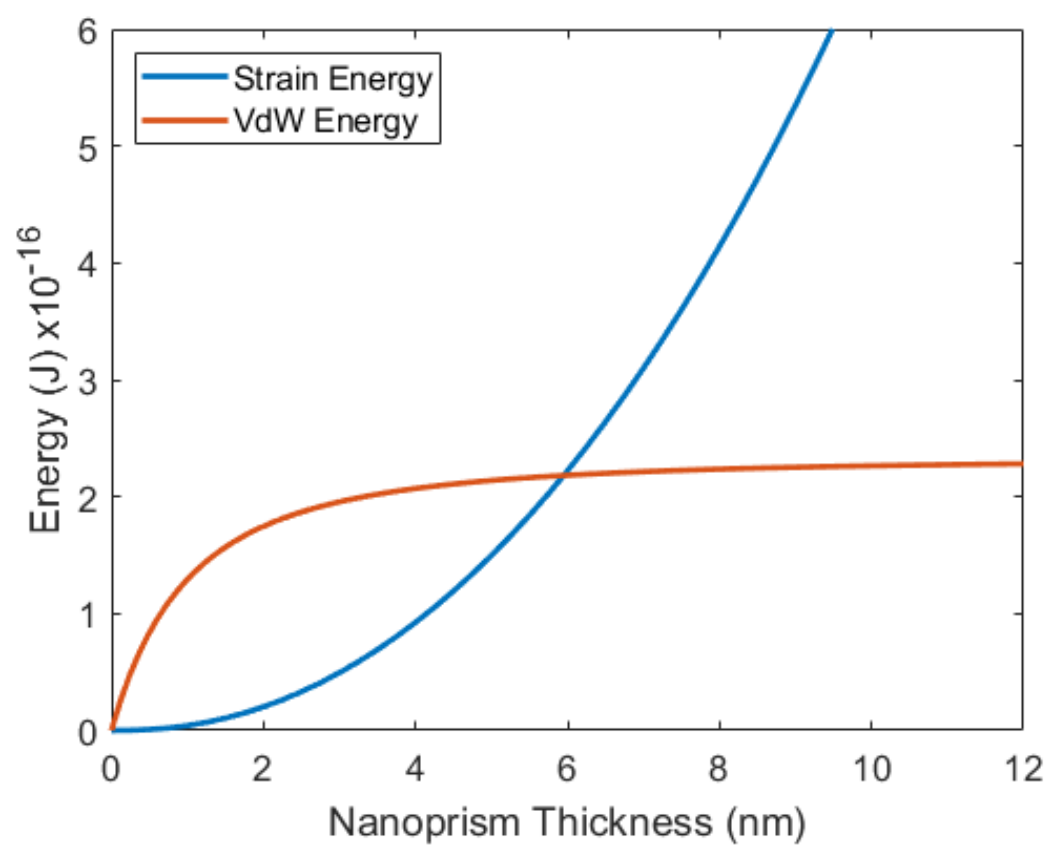

Figure S11. Plot of strain energy and VdW energy against nanoplate thickness. $W_{\max }=30 \mathrm{~nm}, a=50 \mathrm{~nm}, \mathrm{~d}=2 \mathrm{~nm}$ 


\section{Applicability of Theory to Other Materials}

This theory can be applied to other materials if valid material properties can be obtained for them. As a demonstration we carried out the calculation for $\mathrm{SiO}_{2}\left(\sigma_{y}=155 \mathrm{MPa}, \mathrm{v}=0.19, \mathrm{E}\right.$ $=74.8 \mathrm{GPa}, A_{\mathrm{SiO}_{2}}=6.55 \times 10^{-20} \mathrm{~J}[6,13]$. This is shown in Fig. S12 which shows that for the same degree of deformation as previous examples, an $\mathrm{SiO}_{2}$ nanoplate would need to be thinner.

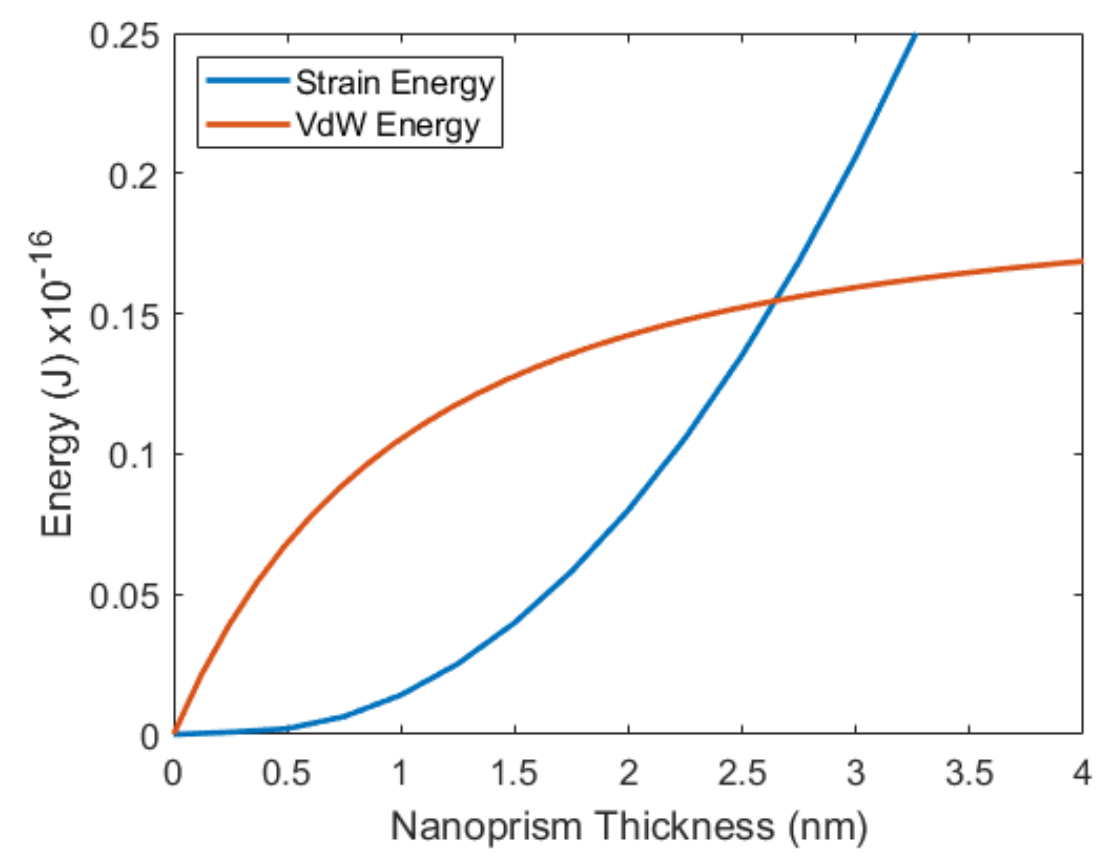

Figure S12. Plot of strain energy and $\mathrm{VdW}$ energy against $\mathrm{SiO}_{2}$ nanoplate thickness. $W_{\max }=15 \mathrm{~nm}, a=50 \mathrm{~nm}, \mathrm{~d}=2 \mathrm{~nm}$

Another example is $\mathrm{CdSe}\left(\sigma_{y}=8.55 \mathrm{GPa}, \mathrm{v}=0.345, \mathrm{E}=61.2 \mathrm{GPa}, A_{C d S e}=6.208 \times 10^{-20} \mathrm{~J}\right)$

[15],[16]. This is shown in Fig. S13, which shows that a CdSe nanoplate would need to be less $<$ $1 \mathrm{~nm}$ in thickness to be deformed by VdW forces according to the input parameters used. Incidentally, CdSe nanosheets of $0.9 \mathrm{~nm}$ in thickness have previously been synthesised and display flexibility [17]. 


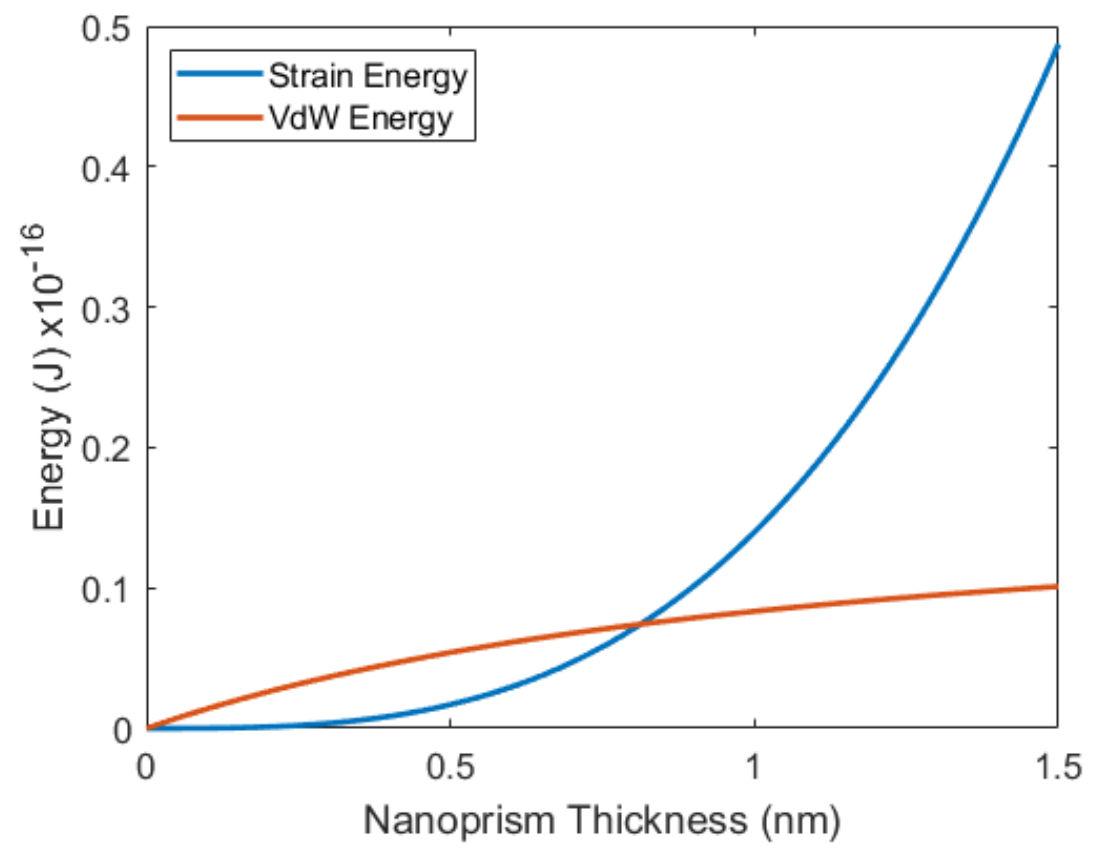

Figure S13. Plot of strain energy and VdW energy against CdSe nanoplate thickness. Wmax $=15 \mathrm{~nm}, a=50 \mathrm{~nm}, \mathrm{~d}=2 \mathrm{~nm}$.

\section{FEM and Solid Mechanics Calculations}

The analytical Kirchhoff-Love theory used in this work and described in detail above is strictly applicable when plate deformations are small compared to the plate thickness $t$. In addition, our analytical modeling also assumes that the sphere-plate contact is approximated as a point load at $r=0$. We verified that these two assumptions provide reasonably accurate models for our experiments by using steady-state finite-element method (FEM) calculations (COMSOL Multiphysics with the Nonlinear Solid Mechanics module) to determine the axisymmetric deformations of a silver nanoplate around an iron oxide template particle. The FEM simulations do not assume that deformations are small, and do not assume a point contact. To facilitate comparison with the Kirchhoff-Love theory that leads to the displacement field of Eq. (1), vander-Waals forces are not included in the simulation, and both materials in the calculation are taken to be elastic, homogeneous, and isotropic. Note that the assumption of elastic deformation is expected to fail because significant plastic deformation occurs due to stresses exceeding the yield 
stress, as discussed above. Here, we consider only elastic deformation to focus on the comparison with basic Kirchhoff-Love theory; the unrealistically large stresses observed in the simulation results indicate that plastic deformation occurs in the experiment.

In the FEM calculations, the nanoplate has an initial (i.e. undeformed) thickness of $t_{0}=$ $9 \mathrm{~nm}$, and the iron oxide sphere has an initial diameter of $d_{0}=15 \mathrm{~nm}$. The nanoplate is clamped at the points $(r, z)=(a, 0)$ and $\left(a, t_{0}\right)$. The iron oxide particle rests below the nanoplate in an undeformed state. To initiate deformation, the sphere is displaced in the axial $z$ direction with a prescribed displacement $w=d_{0}$ for all points on the surface of the sphere. After this displacement, the bottom point on the sphere lies in the same $z=0$ plane as the clamped bottom edge of the plate. This configuration represents the experimental scenario where both the template particle and the clamped portions of the nanoplate are supported by the substrate at the $z=0$ plane. Note that the substrate itself is not necessary to include in our FEM models because the $\mathrm{VdW}$ forces are not considered, and the nanoplate is clamped at $r=a$.

The contact between the sphere and nanoplate is calculated in a self-consistent manner along with the displacements, stresses, and strains in both materials. The contact surface is assumed to be frictionless, and no adhesion forces are considered. In the scenario where displacements at the contact are sufficiently small, our FEM contact modelling reproduces the results of classic Hertzian contact theory; deviations from Hertzian theory arise when the contact radius is not negligibly small compared to $d_{0}$. To avoid numerical difficulties associated with the nonlinearities in contact mechanics problems involving large displacements, we employ parametric sweeps over different template particle displacements and ensure that all results are converged with respect to the spatial mesh.

To compare the deflection fields against the experimental results and to generate stress profiles to understand the mechanical stress induced by the deformation, we performed several simulations of the experimental geometry in which a sphere deforms a clamped nanoplate disc. Also, for each simulation we have characterized the contact radius between the sphere and the 
nanoplate; we found that the value of the contact radius stays relatively stable across different geometric and material parameters. Additionally, to prove that VdW forces are necessary to achieve the deformed structures we observe experimentally, we also modelled the same sphere deforming a large triangular nanoplate, clamped at the edges. For every configuration we performed simulations using both the bulk Young's modulus, $74 \mathrm{GPa}$, as well as an elevated Young's modulus, $125 \mathrm{GPa}$, a phenomenon that is typically associated with nano-silver. Note that in all cases, the stresses required to achieve deformation exceed even an elevated yield stress value for nano-silver $(\sim 1 \mathrm{GPa})$, indicating the plastic deformation would occur in the experiment.

$\underline{\text { Simulations of a } 75 \mathrm{~nm} \text { Radius Disc }}$
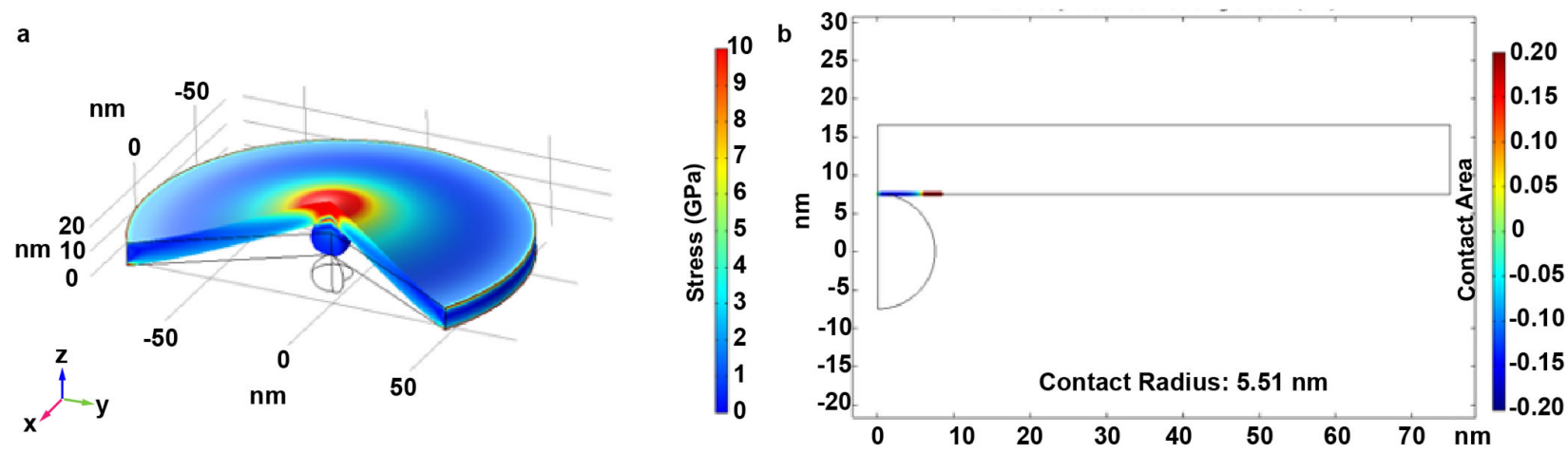

Figure S14. Simulation of clamped disc with bulk Young's Modulus, 74 GPa. (a) Surface stress map of deformed clamped disc and (b) plot showing the contact radius between the sphere and disc.
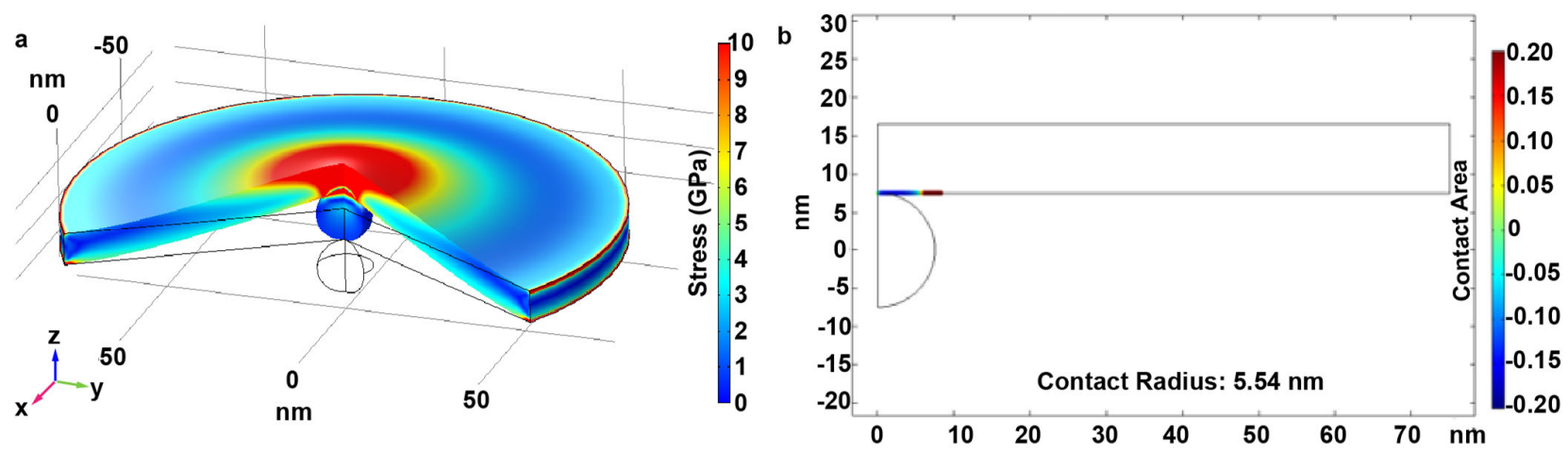

Figure S15. Simulation of clamped disc with elevated Young's Modulus, 125 GPa. (a) Surface stress map of deformed clamped disc and (b) plot showing the contact radius between the sphere and disc. Panel (a) can be found as a component of Fig. 3 in the main text. 
$\underline{\text { Simulations of a } 50 \mathrm{~nm} \text { Radius Disc }}$

*Note that for this set of simulations, the maximum displacement is $10 \mathrm{~nm}$. Results for higher displacement values would not converge in COMSOL, due to the large stresses and strains.
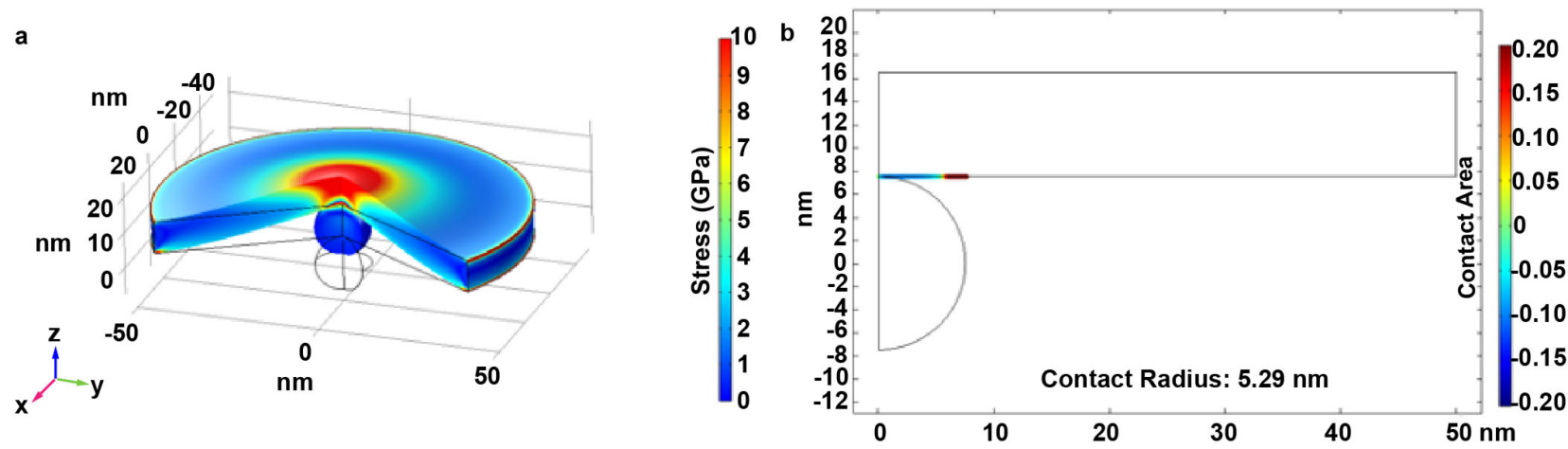

Figure S16. Simulation of clamped disc with bulk Young's Modulus, 74 GPa. (a) Surface stress map of deformed clamped disc and (b) plot showing the contact radius between the sphere and disc.
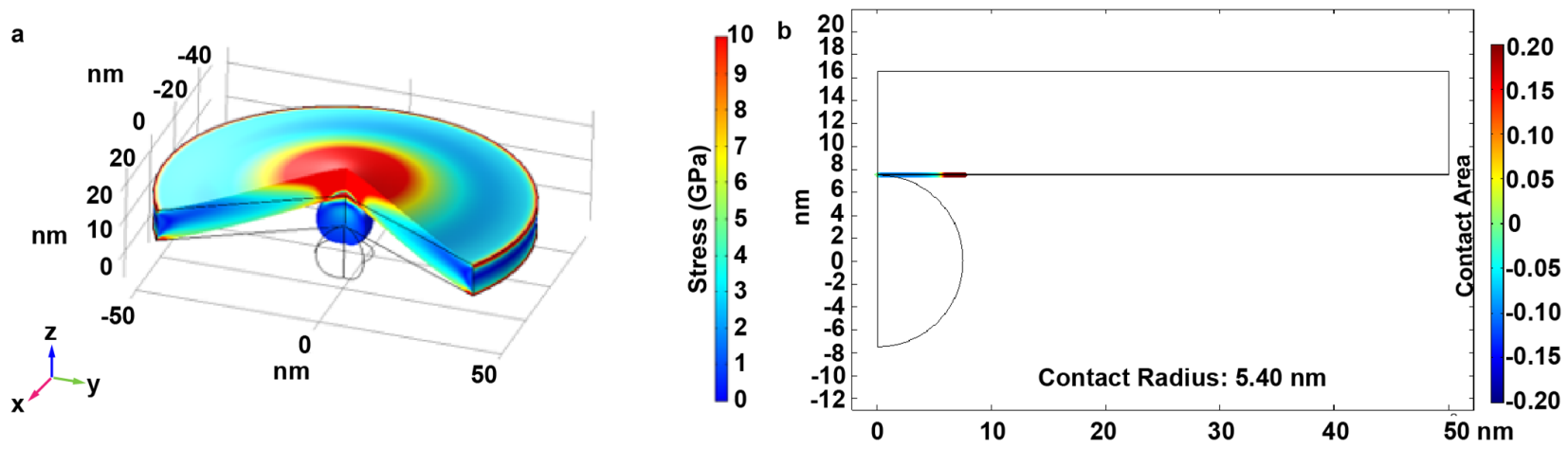

Figure S17. Simulation of clamped disc with elevated Young's Modulus, 125 GPa. (a) Surface stress map of deformed clamped disc and (b) plot showing the contact radius between the sphere and disc. 


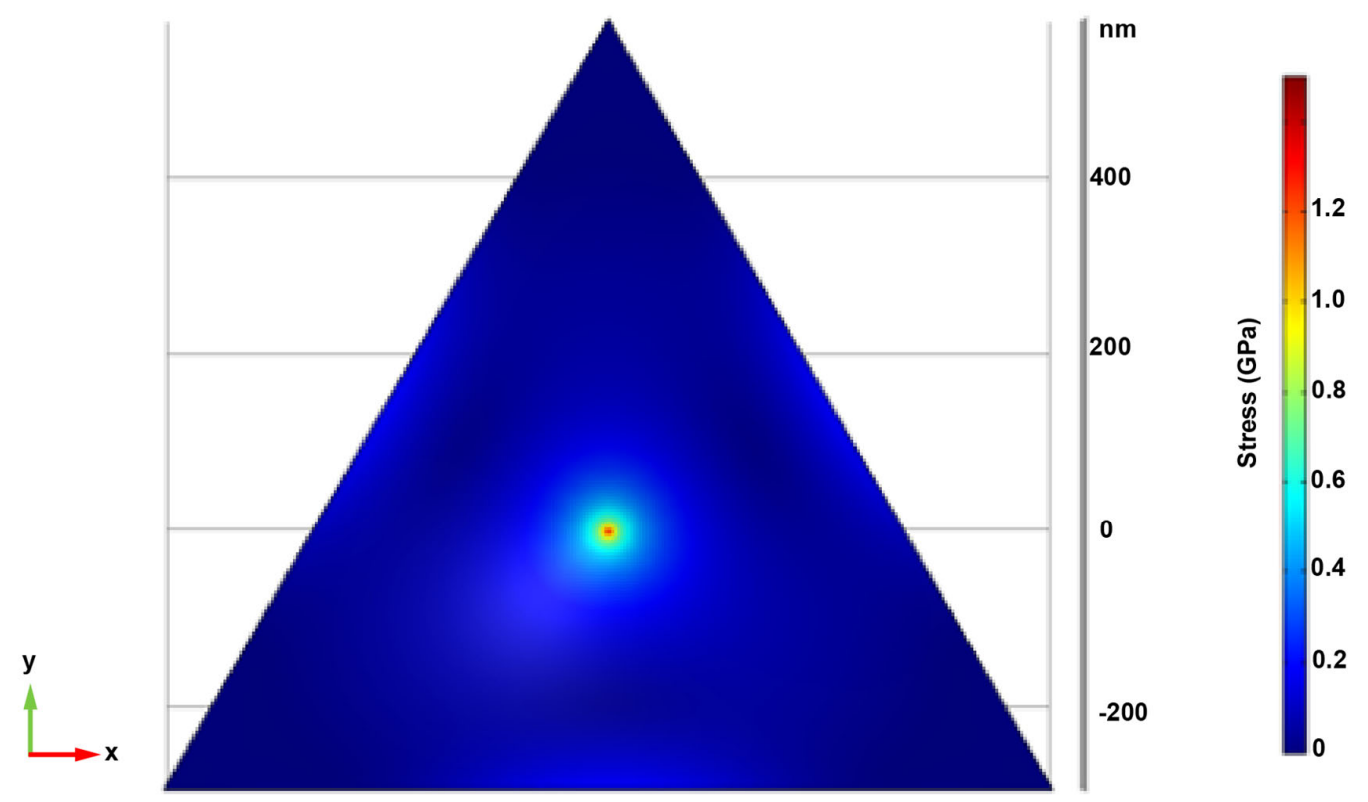

Figure S18. Surface stress map of clamped nanoplate with bulk Young's Modulus, 74 GPa.

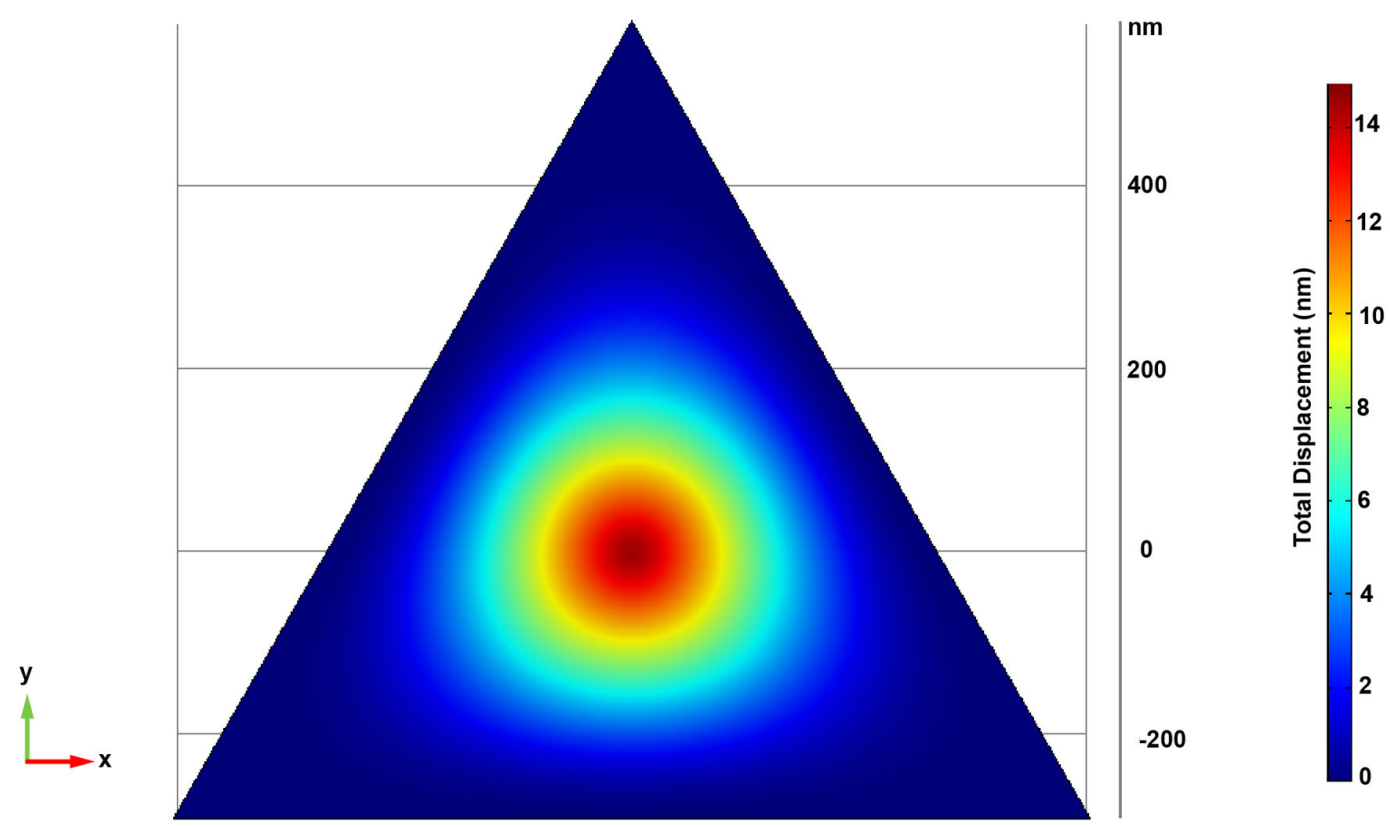

Figure S19. Total displacement map of clamped nanoplate with bulk Young's Modulus, 74 GPa. 
$\underline{\text { Simulations of a } 1 \text { um Edge-Length Nanoplate for Elevated Young's Modulus Values }}$

*Note that for this set of simulations, the maximum displacement is $10 \mathrm{~nm}$. Results for higher displacement values would not converge in COMSOL.

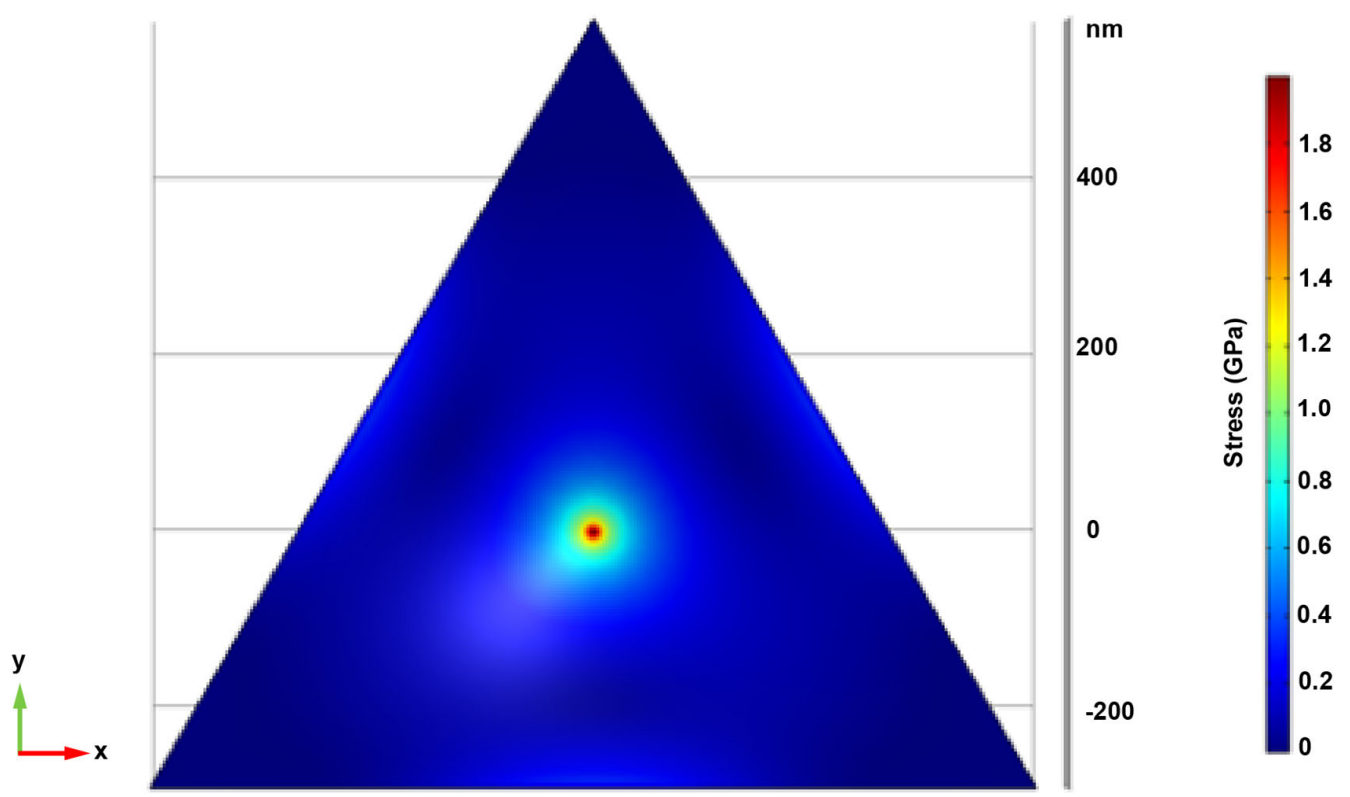

Figure S20. Surface stress map of clamped nanoplate with elevated Young's Modulus, 125 GPa.

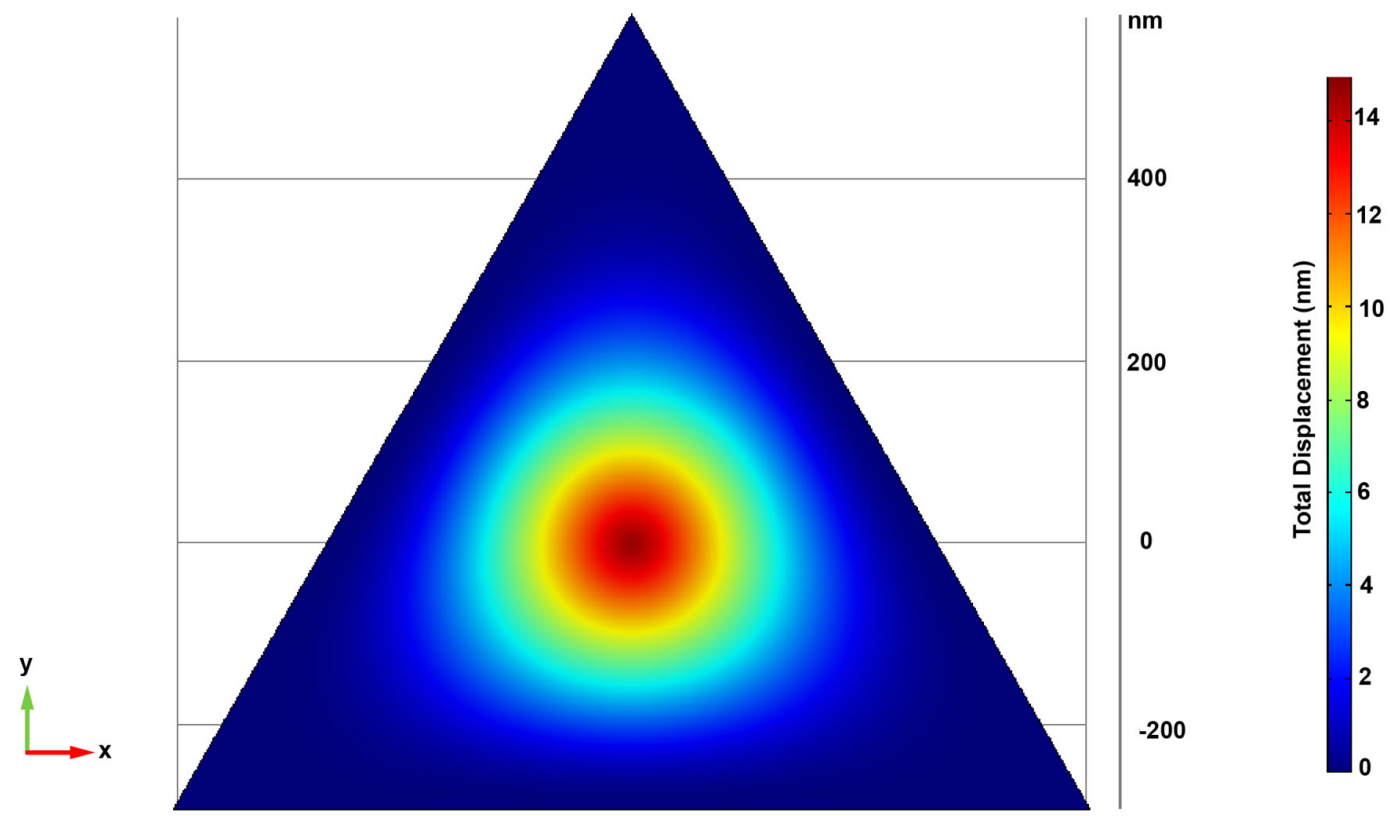

Figure S21. Total displacement map of clamped nanoplate with bulk Young's Modulus, 74 GPa. 


\section{References}

[1] Zhang, Q.; Li, N.; Goebl, J.; Lu, Z.; Yin, Y. J. Am. Chem. Soc. 2011, 133, 18931-18939.

[2] Liu, X.; Li, L.; Yang, Y.; Yin, Y.; Gao, C. Nanoscale 2014, 6, 4513-4516.

[3] Sutti, M. Elastic Theory of Plates, École Polytechnique Fédérale de Lausanne, Switzerland, May 2015. http://www.unige.ch/math/folks/sutti/Elastic Theory of Plates.pdf

[4] Eraslan, A. N.; Argeso, H. Turkish J. Eng. Env. Sci. 2005, 29, 113-128.

[5] Tadmor, R. J. Phys.: Condens. Matter 2001, 13, L195-L202.

[6] Leite, F. L.; Bueno, C. C.; Da Róz, A. L.; Ziemath, E. C.; Oliveira, O. N. Int. J. Mol. Sci. 2012, 13, 12773-12856.

[7] Wu, B.; Heidelberg, A.; Boland, J. J. Nano Lett. 2006, 6, 468-472.

[8] Zhang, S. B. Comput. Mater. Sci. 2014, 95, 53-62.

[9] Gao, Y.; Fu, Y.; Sun, W.; Sun, Y.; Wang, H.; Wang, F.; Zhao, J. Comput. Mater. Sci. 2012, 55, 322328.

[10] Vlassov, S.; Polyakov, B.; Dorogin, L. M.; Antsov, M.; Mets, M.; Umalas, M.; Saar, R.; Lõhmus, R.; Kink, I. Mater. Chem. Phys. 2014, 143, 1026-1031.

[11] Li, X.; Gao, H.; Murphy, C. J.; Caswell, K. K. Nano Lett. 2003, 3, 1495-1498.

[12] Kiani, M. T.; Patil, R. P.; Gu, X. W. MRS Commun. 2019, 9, 1029-1033.

[13] https://www.azom.com/properties.aspx?ArticleID=1114

[14] Lahiri, J.; Isaacs, L.; Tien, J.; Whitesides, G. Anal. Chem. 1999, 71, 777-790.

[15] Chowdhury, E.H.; Rahman, H.; Jayan, R.; Islam, M. Comput. Mater. Sci. 2021, 186, 110001.

[16] Deligoz, E.; Colakoglu, K.; Ciftci, Y. Physica B Condens. Matter 2006, 373, 124-130.

[17] Bouet, C.; Mahler, B.; Nadal, B.; Abecassis, B.; Tessier, M.; Ithurria, S.; Xu, X.; Dubertret, B.

Chem. Mater. 2013, 25,4, 639-645. 